\title{
Learning Curve for Endoscopic Endonasal Dacryocystorhinostomy
}

\author{
Jong Joo Lee, Han Min Lee ${ }^{1}$, Hyung Bin Lim ${ }^{1,2}$, Seong Wook Seo ${ }^{3}$, Hee Bae Ahn ${ }^{4}$, Sung Bok Lee \\ ${ }^{I}$ Department of Ophthalmology, Chungnam National University College of Medicine, Daejeon, Korea \\ ${ }^{2}$ Department of Ophthalmology, Armed Forces Capital Hospital, Seongnam, Korea \\ ${ }^{3}$ Department of Ophthalmology, Gyeongsang National University College of Medicine, Jinju, Korea \\ ${ }^{4}$ Department of Ophthalmology, Dong-A University College of Medicine, Busan, Korea
}

Purpose: To elucidate the learning curve for endoscopic endonasal dacryocystorhinostomy (EE-DCR) based on the results of EE-DCR performed by three surgeons at three different tertiary hospitals.

Methods: A retrospective review of the medical records of 386 eyes of 337 patients who had undergone EEDCR by three surgeons at three tertiary hospitals and who were available for a $>6$-month postoperative observation period was conducted. The success of a given surgery was determined based on the results of a test performed during the patient's last outpatient visit to the hospital. The learning curve was identified by dividing the patients into four groups (20,30,40, and 50 eyes in each respective group) and comparing their success rates.

Results: The overall success rate of the entire study population was $86.3 \%$. The success rates for each of three surgeons was $83.3 \%, 85.6 \%$, and $88.1 \%$, respectively. After dividing the patients into groups of 30 eyes each, all three surgeons showed a significant increase in surgery success rates after their first group of 30 eyes $(p<0.05)$. The overall success rate excluding the first 30 eyes was $92.9 \%$, and all three surgeons exhibited a significantly improved success rate of $>90 \%$ (A, 94.4\%; B, 90.8\%; C, 95.4\%).

Conclusions: A surgeon should be required to perform at least $30 \mathrm{EE}-\mathrm{DCR}$ procedures to obtain stable surgical skill for this procedure.

Key Words: Dacryocystorhinostomy, Lacrimal duct obstruction, Learning curve

Dacryocystorhinostomy (DCR) is the most widely performed surgical procedure for the treatment of epiphora due to nasolacrimal duct obstruction. DCR is typically divided into endonasal DCR and external DCR. Endonasal DCR was first introduced in 1893 by Caldwell [1], but exhibited a low success rate because of limited surgical visibility. Since the introduction of endoscopic endonasal DCR

Received: August 8, 2016 Accepted: November 16, 2016

Corresponding Author: Sung Bok Lee, MD, PhD. Department of Ophthalmology, Chungnam National University Hospital, \#282 Munhwaro, Jung-gu, Daejeon 35015, Korea. Tel: 82-42-280-7604, Fax: 82-42-2553745, E-mail: sblee@cnu.ac.kr
(EE-DCR) in 1989 by McDonogh and Meiring [2], the success rate has dramatically increased to $80 \%$ to $95 \%$, along with the advent of medical devices such as endoscopes and illuminators and the development of endoscopic skills $[3,4]$. In addition, in contrast to external DCR, EE-DCR produces no skin scars, ensures a quick recovery, and has a low occurrence rate of complications, including medial canthal injuries [5-7].

The factors that most influence the success rate of EEDCR reportedly include anatomical factors $[8,9]$ such as the size of the lacrimal sac and nasal cavity, the presence of nasal septum deviation, the presence of middle turbinate hypertrophy, and the surgeon's skill level [10]. Among 
these influential factors, the latter is the only one that can be improved. A high level of skill is needed to achieve a high success rate for EE-DCR because unlike other ocular surgeries, EE-DCR requires a thorough understanding of the anatomical structures inside the nose as well as the skilled use of surgical devices such as endoscopes. However, most ophthalmologists are not familiar with such devices. Although the learning curve for endonasal DCR without endoscopy has been previously described [11], we found no reports on the learning curve for EE-DCR. Therefore, we conducted the present study to elucidate the learning curve for EE-DCR based on the surgical outcomes of EE-DCR performed by three ophthalmic plastic surgery specialists.

\section{Materials and Methods}

\section{Subjects}

This study protocol was approved by the institutional review board of each Hospital (Chungnam National University Hospital, Gyeongsang National University Hospital, and Dong-A University Hospital). The study adhered to the tenets of the Declaration of Helsinki. A retrospective review of the medical records of patients who had undergone EE-DCR from January 2001 to February 2013 by one of three ophthalmic plastic surgery specialists and who were available for a $>6$-month postoperative observation period was conducted. All patient records and information was anonymized and de-identified prior to analysis. None of the three surgeons learned how to perform EE-DCR through official oculoplastic fellowship training, and the first and all following cases performed by each surgeon during their early surgical experiences were included in this study.

Preoperative slit lamp examination, lacrimal irrigation, and dacryocystography were performed in all patients, and then nasal endoscopy was conducted to evaluate their nasal cavities. Patients were diagnosed with nasolacrimal duct obstruction when subjective symptoms of epiphora were found, if fluid passed into the nose with resistance and reflux occurred during the lacrimal irrigation procedure, or when a partial or complete blockage of the nasolacrimal duct below the lacrimal sac was observed. Patients with a history of trauma-induced epiphora, tumor- induced nasolacrimal duct obstruction, common canalicular obstruction or stenosis, or those with a history of DCR were excluded from the study.

\section{Surgical techniques}

All surgeries were performed by three surgeons under general anesthesia using a standard DCR procedure. Each surgeon enlarged the bony ostium via proper use of a Kerrison punch or drill. A vertical incision was created in the lacrimal sac using a keratome, sickle knife, or laser, and the medial wall of the sac was excised. In all patients, a silicone tube was passed through both the upper and lower puncta and a knot was tied in the nasal cavity. Surgery was completed by packing Vaseline gauze or Merocel soaked in antibiotic eye ointment via an intranasal route. Following surgery, all patients were given a topical steroid, antibiotic eye drops for administration three times a day, and a steroid nasal spray (budesonide) for administration once a day. The silicone tube was removed 2 to 6 months later, depending on the patient's condition.

The surgery was deemed successful if a lacrimal irrigation test exhibited free flow and patency with no complications, such as adhesion or stenosis of the internal lacrimal opening, as observed endoscopically at the patient's last clinic visit.

\section{Statistical analysis}

All statistical analyses were performed using PASW for Windows ver. 18.0 (SPSS Inc., Chicago, IL, USA). Patients' clinical findings and procedural details were compared among the three surgeons using analysis of variance. The turning point of the DCR learning curve can be defined as the time at which there is a significant increase in the surgical success rate of endoscopic DCR compared to the previous procedure and after which a higher DCR success rate is maintained. In the present study, we calculated the success rate of endoscopic DCR in each group by dividing the total population into groups of $20,30,40$, and 50 eyes each in sequential order to determine the turning point of the DCR learning curve. We also made every effort to calculate the minimum number of surgeries to ensure that the success rate of EE-DCR significantly increased and was continuously maintained when the success rate was compared among the three surgeons. We repeated comparisons 
of the success rate by categorizing the patients into those who underwent the procedure before and after the minimum number of surgeries to ascertain whether the minimum number of surgeries was equivalent to the turning point of the DCR learning curve. The success rates of the three surgeons were compared using a chi-square test. Statistical significance was set at a $p$-value $<0.05$.

\section{Results}

In total, 386 eyes of 337 patients were included in this study; 58 patients were male and 337 patients were female. Surgeon A operated on 66 eyes of 60 patients; surgeon B operated on 160 eyes of 144 patients; and surgeon $C$ operated on 160 eyes of 133 patients. The average patient age was $54.4 \pm 14.3$ years (surgeon A, $55.9 \pm 13.8$ years; surgeon B, $55.4 \pm 13.9$ years; surgeon C, $52.7 \pm 14.3$ years). The average observation period was $10.0 \pm 9.3$ months (surgeon A, $7.5 \pm 7.0$ months; surgeon B, $9.5 \pm 10.2$ months; surgeon $C, 8.9 \pm 16.0$ months). There were no significant differences in age, sex, or observation period among the patient groups of the three surgeons $(p>0.05)$ (Table 1).

The overall surgery success rate of the entire study population was $86.3 \%(333 / 386)$. The success rates of the right and left eyes were $83.7 \%$ (170 / 203) and 88.5\% (162/ $183)$, respectively, with no statistically significant difference between them.

With respect to the surgical success rate of each surgeon, surgeons A, B, and C were successful in 55 / 66 eyes (83.3\%), $137 / 160$ eyes (85.6\%), and $141 / 160$ eyes (88.1\%), respectively. These results were not significantly different $(p>0.05)$ (Table 2). The three surgeons performed all surgeries based on the standard DCR procedure, but there were differences in the creation of a large bony ostium and the incision of the lacrimal sac. The different surgical instruments used for creation of the bony ostium are as follows: surgeons $\mathrm{A}$ and $\mathrm{C}$ used a surgical drill and rongeur for all patients, while surgeon B used a rongeur with or without a surgical drill. Furthermore, during incision of the lacrimal sac, surgeons $\mathrm{A}$ and $\mathrm{C}$ performed laser-assisted ablation in all patients, while surgeon B used a knife. Following surgery, surgeons $\mathrm{B}$ and $\mathrm{C}$ gave all of their patients mitomycin $\mathrm{C}$ eye drops (Table 2).

The success rates of the surgeries performed on the first group of 20 eyes by surgeons A, B, and C were $80.0 \%$ (16 / 20 ), 65.0\% (13/20), and 50.0\% (10/20), respectively (Fig. 1A). The success rates of the surgeries performed on the second group of 20 eyes by surgeons A, B, and C were 70.0\% (14 / 20), 80.0\% (16 / 20), and 60.0\% (12/20), respectively; no significant improvement was shown $(p>$ $0.05)$. The success rates of the surgeries performed on the third group of 20 eyes by surgeons A, B, and C were 95.0\% (19 / 20), 80.0\% (16/20), and 95.0\% (19/20), respectively; surgeons $\mathrm{A}$ and $\mathrm{C}$ exhibited a significant increase in their success rates. The subsequent groups of 20 eyes showed a steady increase in the success rate, but none of them revealed a significant improvement.

The success rates of the surgeries performed on the first group of 30 eyes by surgeons A, B, and C were $70.0 \%$ (21/ 30), 63.3\% (19 / 30), and 56.7\% (17 / 30), respectively (Fig. 1B). The success rates of the surgeries performed on the second group of 30 eyes by surgeons $\mathrm{A}, \mathrm{B}$, and $\mathrm{C}$ were 93.3\% (28 / 30), 86.7\% (26 / 30), and 86.7\% (26/30), and all three surgeons showed substantially high success rates ( $p=0.020,0.037$, and 0.024, respectively). Surgeons B and $\mathrm{C}$ subsequently maintained a high success rate.

For the groups of 40 and 50 eyes, only surgeons B and C were compared. The success rates of the surgeries performed on the first group of 40 eyes by surgeons B and C

Table 1. Patients' baseline characteristics

\begin{tabular}{lccccc}
\hline & Surgeon A & Surgeon B & Surgeon C & Total & $p$-value \\
\hline No. of eyes (patients) & $66(60)$ & $160(144)$ & $160(133)$ & $386(337)$ & - \\
Age (yr) & $55.9 \pm 13.8$ & $55.4 \pm 13.9$ & $52.7 \pm 14.3$ & $54.4 \pm 14.3$ & $0.943^{*}$ \\
Sex (male: female) & $7: 53$ & $24: 120$ & $27: 106$ & $58: 279$ & $0.330^{\dagger}$ \\
Laterality (RT: LT) & $34: 32$ & $84: 76$ & $85: 75$ & $203: 183$ & $0.800^{\dagger}$ \\
Follow-up period (mon) & $7.5 \pm 7.0$ & $9.5 \pm 10.2$ & $8.9 \pm 16.0$ & $10.0 \pm 9.3$ & $0.552^{*}$ \\
\hline
\end{tabular}

Values are presented as number or mean \pm standard deviation.

$\mathrm{RT}=$ right; $\mathrm{LT}=$ left.

${ }^{*}$ Analysis of variance; ${ }^{\dagger}$ Chi-square test. 
Table 2. Procedural details among the three surgeons

\begin{tabular}{|c|c|c|c|c|c|}
\hline & Surgeon A & Surgeon B & Surgeon C & Total & $p$-value \\
\hline Success rate $(\%)$ & 83.3 & 85.6 & 88.1 & 86.3 & $0.391^{*}$ \\
\hline \multicolumn{6}{|l|}{ Ostectomy device } \\
\hline Drill and rongeur & $66(100.0)$ & $112(70.0)$ & $160(100.0)$ & $338(84.9)$ & $<0.001^{*}$ \\
\hline Rongeur only & 0 & $48(30.0)$ & 0 & $204(52.8)$ & \\
\hline Sac incision & & & & & - \\
\hline Laser & $66(100.0)$ & 0 & $160(100.0)$ & $224(58.1)$ & \\
\hline Knife & 0 & $160(100.0)$ & 0 & $158(40.9)$ & \\
\hline Postoperative management & & & & & - \\
\hline Antibiotics eye drop use & $66(100.0)$ & $160(100.0)$ & $160(100.0)$ & $386(100.0)$ & \\
\hline Steroid eye drop use & $66(100.0)$ & $160(100.0)$ & $160(100.0)$ & $386(100.0)$ & \\
\hline Mitomycin $\mathrm{C}$ eye drop use & 0 & $160(100.0)$ & $160(100.0)$ & $320(82.9)$ & \\
\hline Nasal spray & $66(100.0)$ & $160(100.0)$ & $160(100.0)$ & $386(100.0)$ & \\
\hline Silicone tube intubation & $66(100.0)$ & $160(100.0)$ & $160(100.0)$ & $386(100.0)$ & - \\
\hline Silicone tube removal (mon) & $4.1 \pm 2.5$ & $2.4 \pm 0.9$ & $2.7 \pm 2.4$ & $2.6 \pm 1.5$ & $<0.001^{\dagger}$ \\
\hline
\end{tabular}

Values are presented as number $(\%)$ or mean \pm standard deviation unless otherwise indicated.

${ }^{*}$ Chi-square test; ${ }^{\dagger}$ Analysis of variance.
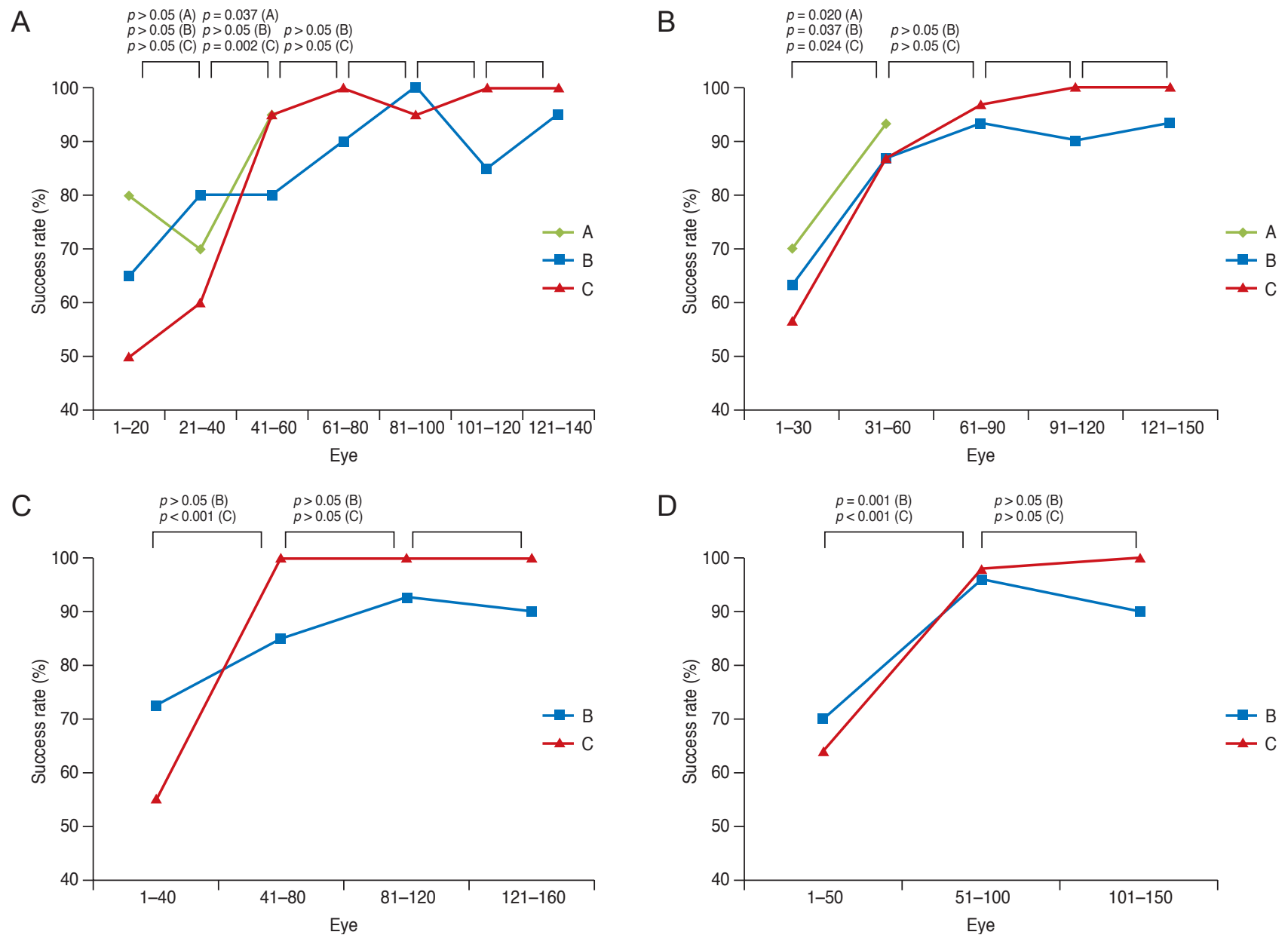

Fig. 1. Changes in the success rate of each consecutive group of 20 (A), 30 (B), 40 (C), and 50 (D) eyes. 
were $72.5 \%$ (29 / 40) and 56.7\% (22 / 40), respectively (Fig. 1C). The success rates of the surgeries performed on the second group of 40 eyes by surgeons $\mathrm{B}$ and $\mathrm{C}$ were $85.0 \%$ (34 / 40) and 100.0\% (40/40), respectively; surgeon C showed a more improved success rate than surgeon $\mathrm{B}(p<$ 0.001 and $p=0.172$ ). Finally, surgeons $\mathrm{B}$ and $\mathrm{C}$ exhibited significantly greater success rates for the second group than for the first group of 50 eyes ( $p=0.001$ and $p<0.001$, respectively) (Fig. 1D).

The success rates of EE-DCR among the three surgeons were compared by dividing the eyes into two groups of 30 eyes each (i.e., the success rates before and after 30 eyes were compared). Before surgery on the 30 eyes, the success rates achieved by surgeons $\mathrm{A}, \mathrm{B}$, and $\mathrm{C}$ were $70.0 \%$ (21/ 30), 63.3\% (19 / 30), and 56.7\% (17 / 30), respectively; however, after performing surgery on $>30$ eyes, the success rates significantly increased. The success rates achieved by surgeons A, B, and C were 94.4\% (34 / 36), 90.8\% (118 / 130), and $95.4 \%$ (124 / 130), respectively; all changes were statistically significant (Table 3 ).

\section{Discussion}

DCR is the most widely practiced surgical procedure for treating nasolacrimal duct obstruction. In particular, the apparent advantages of endonasal over external DCR include a lack of cutaneous scarring, a minimal risk of bleeding, early return to daily life secondary to less discomfort and rapid recovery after surgery, and preservation of the pump function of the orbicularis oculi muscle, which remains intact. However, early endonasal DCR has been associated with a lower success rate than external DCR because of the difficulty in obtaining adequate visualization of the operation field. Furthermore, the reported complications of endonasal DCR, such as postoperative membranous obstruction of the bony ostium associated with granulation tissue or restenosis, have been greater in number than those of external DCR due to the failure of direct suturing of the nasal and lacrimal mucosal flaps [2$7,12,13]$.

Many studies have investigated how to improve the success rate of endonasal DCR. Linberg et al. [14] suggested that the creation of a sufficiently sized bony ostium followed by excision of the medial sac wall are important for increasing the success rate of endonasal DCR and reducing the postoperative recurrence rate. Iliff [15] reported that postoperative management, including washing of the lacrimal duct, probing, and removal of the intranasal mucous membrane and granulomas, may affect the success rate of endonasal DCR. Several studies have also revealed that the use of mitomycin C improves the outcome; this is because the antimetabolite both reduces scarring at the bony ostium and surrounding areas and decreases granuloma formation by inhibiting the proliferation of fibroblasts, delaying wound healing, or preventing scarring at the opening [16-18]. Some studies have reported that postoperative use of steroid nasal spray may improve the success rate of endonasal DCR by inhibiting anti-inflammatory action and granuloma formation [13].

With the advancement of nasal endoscopes and surgical instruments for creation of the bony ostium, EE-DCR has recently shown higher success rates of $80 \%$ to $95 \%$, similar to that of external DCR. EE-DCR is the most widely accepted procedure for correcting the acquired nasolacrimal duct obstructions in patients with epiphora [2-7].

Nevertheless, a heavy disadvantage of EE-DCR is that thorough knowledge of the intranasal anatomy is necessary, thus requiring the surgeon to employ a nasal endoscope, which is not used in other ophthalmic surgeries. Thus, inexperienced surgeons may find it difficult to manipulate the instruments in the nasal cavity. High skill lev-

Table 3. Success rates of each group of 30 eyes among the three surgeons

\begin{tabular}{lcccr}
\hline & Eyes 1-30 & Eyes $\geq 31$ & Total & $p$-value \\
\hline Surgeon A & $21 / 30(70.0)$ & $34 / 36(94.4)$ & $55 / 66(83.3)$ & 0.020 \\
Surgeon B & $19 / 30(63.3)$ & $118 / 130(90.8)$ & $137 / 160(85.6)$ & $<0.001$ \\
Surgeon C & $17 / 30(56.7)$ & $124 / 130(95.4)$ & $141 / 160(88.1)$ & $<0.001$ \\
Total & $57 / 90(63.3)$ & $276 / 296(93.2)$ & $333 / 386(86.3)$ & $<0.001$ \\
$p$-value & 0.350 & 0.097 & - & \\
\hline
\end{tabular}

Values are presented as number (\%).

${ }^{*}$ Chi-square test between eyes $1-30$ and eyes $\geq 31 ;{ }^{\dagger}$ Chi-square test among the three surgeons. 
els in endoscopic nasal surgery are more likely to improve the outcome of EE-DCR than are high skill levels in other ophthalmic surgeries.

The turning point of the DCR learning curve commonly refers to the point at which a high surgical success rate is maintained and severe postoperative complications are reduced. After the turning point, the surgeon will have sufficiently mastered the technique. To date, few studies have reported on the learning curve of endonasal DCR. Lee and Chung [10] reported that when the surgical success rate was compared in each group (about 40 surgeries per group) on a 1-year basis, the success rates gradually increased depending on the surgical period, and a success rate of $92.3 \%$ was achieved 2 years postoperatively. Gu et al. [11] reported that when the surgical success rate was compared in each group (about 50 surgeries per group) on an 8-month basis, the surgical success rate was stabilized from the second group onward, implying that a novice surgeon should perform at least 50 surgeries to ensure a high DCR success rate. To the best of our knowledge, however, the learning curve for EE-DCR has yet to be reported.

In the present study, we compared the number of surgeries performed in each group regardless of the surgery period in order to determine the number of surgeries necessary to ensure proper knowledge of the technique, unlike the above-mentioned reports. The minimum number of surgeries with a significant success rate was 30 eyes, which was the case for all three surgeons (Fig. 1). Based on these results, we compared the surgical success rate between the first group (30 eyes) and the second group ( $\geq 31$ eyes). The surgical success rate of each surgeon was lower than the total surgical success rate until EE-DCR was performed on the first 30 eyes; the success rates of surgeons A, B, and $\mathrm{C}$ were $70.0 \%$ (21 / 30 eyes), 63.3\% (19/ 30 eyes), and $56.7 \%$ (17 / 30 eyes), respectively. However, there was an increasing trend in the surgical success rate from $\geq 31$ eyes onward (Table 3). After performing surgeries on $\geq 31$ eyes, the total surgical success rate of $93.6 \%$ (surgeon A, $94.4 \%$; surgeon B, 90.8\%; surgeon C, 95.4\%) was similar to that in existing reports on EE-DCR and external DCR. Therefore, a novice surgeon should perform EE-DCR on at least 30 eyes until he or she is able to master the necessary surgical skills, including use of an endoscope to obtain a broader visualization of the operation site; this ensures that a high surgical success rate can be maintained.

This study has several limitations. First, endoscopic
DCR was performed using different surgical instruments preferred by each surgeon, each surgery had different durations of silicone intubation, and there were different preoperative patient conditions. These various factors can influence the success rate of EE-DCR. We assumed that a patient's conditions, including age and nasal cavity anatomy, would be similar for all three surgeons. The point of this study is to determine how many surgical cases are needed to become accustomed to an unfamiliar nasal cavity surgery using an unfamiliar endoscope. There was no significant differences among the three surgeons for eyes $1-30$ or for $\geq 31$ eyes (Table 3), and a significant increase in the success rate was noted from $\geq 31$ eyes onward for all three surgeons (Fig. 1). Second, because one surgeon performed a smaller number of surgeries than the other two, we had to compare the surgical success rates every 20 or 30 eyes for each surgeon. However, the surgeon was still able to perform the minimum number of surgeries ( 30 eyes) with a high success rate, as was the case for the other two surgeons, and the results were statistically significant. Third, minor technical changes may exist during the study period. However, these minor changes do not seem to be significant because the success rates after 30 surgeries and overall success rates among the surgeons were not significantly different. Last, patient and surgical information was insufficient because this is a retrospective and multicenter study. Therefore, we could not analyze which factors might have an influence on DCR failure. Thus, our data analysis should be verified by further prospective, multicenter studies.

In conclusion, a novice surgeon should perform at least 30 EE-DCR procedures until he or she masters proper operative technique, which ensures a high surgical success rate that can be maintained. In addition, the development of an educational method designed to shorten the learning curve of EE-DCR should be required during residency or fellow training.

\section{Conflict of Interest}

No potential conflict of interest relevant to this article was reported. 


\section{Acknowledgements}

This work was supported by research fund of Chungnam National University.

\section{References}

1. Caldwell GW. Two new operations for obstruction of the nasal duct with preservation of the canaliculi. Am J Ophthalmol 1893;10:b6.

2. McDonogh M, Meiring JH. Endoscopic transnasal dacryocystorhinostomy. J Laryngol Otol 1989;103:585-7.

3. Chong KK, Lai FH, Ho M, et al. Randomized trial on silicone intubation in endoscopic mechanical dacryocystorhinostomy (SEND) for primary nasolacrimal duct obstruction. Ophthalmology 2013;120:2139-45.

4. Christensen AL, Hansen PO, Jorgensen BG, Autzen T. Endoscopic dacryocystorhinostomy seems promising for lacrimal stenosis. Dan Med J 2013;60:A4581.

5. Jin HR, Yeon JY, Choi MY. Endoscopic dacryocystorhinostomy: creation of a large marsupialized lacrimal sac. $J$ Korean Med Sci 2006;21:719-23.

6. Rebeiz EE. Endoscopic dacryocystorhinostomy. Curr Opin Otolaryngol Head Neck Surg 1999;7:44.

7. Onerci M, Orhan M, Ogretmenoglu O, Irkec M. Long-term results and reasons for failure of intranasal endoscopic dacryocystorhinostomy. Acta Otolaryngol 2000;120:319-22.

8. Park JD, Kim YI, Shin SG. The factors related to surgical success rate of endonasal dacryocystorhinostomy. $J$ Korean Ophthalmol Soc 1998;39:2848-53.
9. Lee DP, Yang SW, Choi WC. The relation between nasal cavity size and success rate in endonasal dacryocystorhinostomy. J Korean Ophthalmol Soc 2000;41:1118-23.

10. Lee HC, Chung WS. Success rate of endonasal dacryocystorhinostomy. J Korean Ophthalmol Soc 1996;37:211-8.

11. Gu BY, Kim WJ, Son JH. Learning curve for endonasal dacryocystorhinostomy. J Korean Ophthalmol Soc 2011;52: 519-23.

12. Ibrahim HA, Batterbury M, Banhegyi G, McGalliard J. Endonasal laser dacryocystorhinostomy and external dacryocystorhinostomy outcome profile in a general ophthalmic service unit: a comparative retrospective study. Ophthalmic Surg Lasers 2001;32:220-7.

13. Woog JJ, Kennedy RH, Custer PL, et al. Endonasal dacryocystorhinostomy: a report by the American Academy of Ophthalmology. Ophthalmology 2001;108:2369-77.

14. Linberg JV, Anderson RL, Bumsted RM, Barreras R. Study of intranasal ostium external dacryocystorhinostomy. Arch Ophthalmol 1982;100:1758-62.

15. Iliff CE. A simplified dacryocystorhinostomy: 1954-1970. Arch Ophthalmol 1971;85:586-91.

16. Yeatts RP, Neves RB. Use of mitomycin C in repeat dacryocystorhinostomy. Ophthal Plast Reconstr Surg 1999;15:19-22.

17. Lee TS, Rhee K. The effect of mitomycin-C eyedrop on prevention of internal ostium obstruction after endonasal dacryocystorhinostomy. J Korean Ophthalmol Soc 1998;39: 1915-20.

18. Kim YT, Chung WS. The effect of mitomycin C in endonasal dacryocystorhinostomy. J Korean Ophthalmol Soc 2002;43:728-32. 